\title{
Bitcoin Transaction Malleability and MtGox
}

\author{
Christian Decker and Roger Wattenhofer \\ ETH Zurich, Switzerland \\ cdecker@tik.ee.ethz.ch, wattenhofer@ethz.ch
}

\begin{abstract}
In Bitcoin, transaction malleability describes the fact that the signatures that prove the ownership of bitcoins being transferred in a transaction do not provide any integrity guarantee for the signatures themselves. This allows an attacker to mount a malleability attack in which it intercepts, modifies, and rebroadcasts a transaction, causing the transaction issuer to believe that the original transaction was not confirmed. In February 2014 MtGox, once the largest Bitcoin exchange, closed and filed for bankruptcy claiming that attackers used malleability attacks to drain its accounts. In this work we use traces of the Bitcoin network for over a year preceding the filing to show that, while the problem is real, there was no widespread use of malleability attacks before the closure of MtGox.
\end{abstract}

Keywords: Bitcoin, Transaction, Signature, Malleability, MtGox, Theft.

\section{Introduction}

In recent years Bitcoin [1] has gone from a little experiment by tech enthusiasts to a global phenomenon. The cryptocurrency is seeing a rapid increase in adoption as well as in value. Bitcoin is inching closer to the stated goal of creating a truly decentralized global currency that facilitates international trade.

A major contribution of the success that Bitcoin is having today has to be attributed to the emergence of Bitcoin exchanges. A Bitcoin exchange is a platform that facilitates buying and selling bitcoins for fiat money like US dollars. This enables a larger public to come in contact with bitcoins, increasing their value as a means to pay for goods and services. Exchanges also provide the ground truth for the value of bitcoins by publishing their trade book and allowing market dynamics to find a price for the traded bitcoins. Finally, much of the media attention focuses on the rapid gain in value that these services have enabled.

However, centralized exchanges are also potential points of failure, in a system that is otherwise completely decentralized. Several high value thefts from these services have made the headlines, never failing to predict the impending doom of Bitcoin as a whole. Additionally a small and mostly sentiment driven market, combined with a quick and easy way to buy and sell bitcoins, facilitates flash crashes and rapid rallies for no apparent reason.

The first, and for a long time largest, Bitcoin exchange was MtGox. Founded in 2010 it was a first stop for many early adopters. With the creation of other

M. Kutyłowski and J. Vaidya (Eds.): ESORICS 2014, Part II, LNCS 8713, pp. 313-326, 2014.

(C) Springer International Publishing Switzerland 2014 
exchanges its monopoly slowly faded, but in February 2014 it still accounted for close to $70 \%$ of all bitcoins ever traded. In February 2014 MtGox had to file for bankruptcy and suspend operations following the loss of over 500 million USD worth of bitcoins owned by its customers.

As the principal cause for the loss, MtGox cited a problem in the Bitcoin protocol: transaction malleability. A user could request a withdrawal from MtGox to a Bitcoin address. The exchange would then create a corresponding transaction and publish it to the Bitcoin network. Due to the way MtGox tracked confirmation of these transactions it could be tricked, exploiting transaction malleability, into believing the transaction to have failed even though it was later confirmed by the network. MtGox would then credit the amount back to the user's account. Effectively the user would have doubled the withdrawn bitcoins, once from the withdrawal and once on its account on MtGox.

In this work we investigate two fundamental questions: Is transaction malleability being exploited? And is the claim that it has been used to bring down MtGox plausible?

\section{Transaction Malleability}

The Bitcoin network is a distributed network of computer nodes controlled by a multitude of owners. They collectively implement a replicated ledger that tracks the address balances of all users. Each user may create an arbitrary number of addresses that can be used to send and receive bitcoins. An address is derived from an ECDSA key pair that is later used to prove ownership of the bitcoins associated with that address.

The only operation allowed to modify address balances are transactions. A transaction is a signed data structure that on the one hand claims some bitcoins associated with a sending address and on the other hand reassigns them to receiving addresses. Transactions are identified by the SHA256 hash of their serialized representation. A transaction consists of one or more inputs and an ordered list of one or more outputs. An input is used to specify which bitcoins will be transferred, while an output specifies the address that should be credited with the bitcoins being transferred. Formally, an output is a tuple comprising the value that is to be transferred and a claiming condition, expressed in a simple scripting language. An input includes the hash of a previous transaction, an index, and a claiming script. The hash and index form a reference that uniquely identifies the output to be claimed and the claiming script proves that the user creating the transaction is indeed the owner of the bitcoins being claimed.

\subsection{Bitcoin Scripts}

The scripting language is a, purposefully non-Turing complete, stack-based language that uses single byte opcodes. The use of the scripting language to set up both the claiming conditions and the claiming scripts allows the creation of complex scenarios for the transfer of bitcoins. For example, it is possible to create 
multi-signature addresses that require $m$-of- $n$ signatures to spend the associated bitcoins for arbitration purposes. However, the vast majority of transactions use standard scripts that set up a claiming condition requiring the claiming script to provide a public key matching the address and a valid signature of the current transaction matching the public key. For this reason the standard claiming script is generally referred to as scriptSig (a script encoding a signature), whereas the standard claiming condition is referred to as scriptPubKey (a script requiring a public key and a signature). Figure 1 shows the structure of the standard claiming condition (scriptPubKey) as well as the standard claiming script (scriptSig).

Of particular interest in this work are the OP_PUSHDATA operations which specify a number of following bytes to be pushed as a string on the stack. Depending on the length of the string one of several possible flavors may be used. The simplest is a single byte with value between $0 x 00$ and $0 x 4 b$, also called OP_0 which simply encodes the length of the string in itself. Additionally, three other operations allow pushing data on the stack, namely OP_PUSHDATA1, OP_PUSHDATA2 and OP_PUSHDATA4, each followed by 1, 2 or 4 bytes, respectively, encoding a little endian number of bytes to be read and pushed on the stack.

In order to verify the validity of a transaction $t_{1}$ claiming an output of a previous transaction $t_{0}$ the scriptSig of $t_{1}$ and the scriptPubKey specified in $t_{0}$ are executed back to back, without clearing the stack in between. The scriptSig of $t_{1}$ pushes the signature and the public key on the stack. The scriptPubKey of $t_{0}$ then duplicates the public key (OP_DUP) and replaces the first copy with its RIPEMD160 hash (OP_HASH160), this 20 byte derivative of the public key is also encoded in the address. The address from the scriptPubKey is then pushed on the stack and the two top elements are then tested for equality (OP_EQUALVERIFY). If the hash of the public key and the expected hash match, the script continues, otherwise execution is aborted. Finally, the two elements remaining on the stack, i.e., the signature and the public key, are used to verify that the signature signs $t_{1}$ (OP_CHECKSIG).

Listing 1.1. scriptPubKey

\begin{tabular}{l|}
\hline OP_DUP \\
OP_HASH160 \\
OP_PUSHDATA* \\
<pubKeyHash> \\
OP_EQUALVERIFY \\
OP_CHECKSIG \\
\hline
\end{tabular}

Listing 1.2. scriptSig

$$
\begin{aligned}
& \text { OPPUSHDATA* } \\
& <\text { sig }> \\
& \text { OPPUSHDATA } * \\
& <\text { pubKey }>
\end{aligned}
$$

Fig. 1. The standard claiming condition and claiming script as used by simple transactions transferring bitcoins to an address backed by a single public key

Notice that, although the scriptSigs are attached to the inputs of the transaction, they are not yet known at the time the signature is created. In fact a signature may not sign any data structure containing itself as this would create a circular dependency. For this reason all the claiming scripts are set to a 
script consisting only of a single OP_0 that pushes an empty string on the stack. The user signing the transaction then iterates through the inputs, temporarily replaces the scriptSig field with the corresponding scriptPubKey 1 from the referenced output, and creates a signature for the resulting serialized transaction. The signatures are then collected and inserted at their respective positions before broadcasting the transaction to the network.

The fact that the integrity of the scriptSig cannot be verified by the signature is the source for transaction malleability: the claiming script may be encoded in several different ways that do not directly invalidate the signature itself. A simple example replaces the OP_0 that pushes the public key on the stack with OP_PUSHDATA2 followed by the original length. The claiming script is changed from $0 \mathrm{x} 48<\operatorname{sig}>41<$ pubKey $>$ to $0 \mathrm{x} 4 \mathrm{D} 4800<$ sig $>4 \mathrm{D} 4100<$ pubKey $>$. The encoded signature is valid in both cases but the hash identifying the transaction is different.

Besides these changes in the way pushes are encoded, there are numerous sources of malleability in the claiming script. A Bitcoin Improvement Proposal (BIP) by Wuille 2] identifies the following possible ways to modify the signature and therefore exploit malleability:

1. ECDSA signature malleability: signatures describe points on an elliptic curve. Starting from a signature it is trivial to mathematically derive a second set of parameters encoding the same point on the elliptic curve;

2. Non-DER encoded ECDSA signatures: the cryptographic library used by the Bitcoin Core client, OpenSSL, accepts a multitude of formats besides the standardized DER (Distinguished Encoding Rules) encoding;

3. Extra data pushes: a scriptPubKey may push additional data at the beginning of the script. These are not consumed by the corresponding claiming condition and are left on the stack after script termination;

4. The signature and public key may result from a more complex script that does not directly push them on the stack, but calculates them on the fly, e.g., concatenating two halves of a public key that have been pushed individually;

5. Non-minimal encoding of push operations: as mentioned before there are several options to specify identical pushes of data on the stack;

6. Zero-padded number pushes: excessive padding of strings that are interpreted as numbers;

7. Data ignored by scripts: if data pushed on the stack is ignored by the scriptPubKey, e.g., if the scriptPubKey contains an OP_DROP, the corresponding push in the scriptSig is ignored;

8. Sighash flags can be used to ignore certain parts of a script when signing;

9. Any user with access to the private key may generate an arbitrary number of valid signatures as the ECDSA signing process uses a random number generator to create signatures;

${ }^{1}$ The use of the scriptPubKey in the signed data as placeholder for the scriptSig is likely to avoid collisions. 


\subsection{Malleability Attacks}

One of the problems that Bitcoin sets out to solve is the problem of double spending. If an output is claimed by two or more transactions, these transactions are said to conflict, since only one of them may be valid. A double spending attack is the intentional creation of two conflicting transactions that attempt to spend the same funds in order to defraud a third party.

Research so far has concentrated on a classical version of the double spending attack. An attacker would create two transactions: (1) a transaction that transfers some of its funds once to a vendor accepting bitcoins and (2) a transaction that transfers those same funds back to itself. The goal would then be to convince the vendor that it received the funds, triggering a transfer of goods or services from the vendor to the attacker, and ensuring that the transaction returning the funds to the attacker is later confirmed. This would defraud the vendor as the transfer to the vendor would not be confirmed, yet the attacker received the goods or services.

A malleability attack, while a variant of the double spending attack, is different from the above. The attacker no longer is the party issuing the transaction, instead it is the receiving party. The attacker would cause the victim to create a transaction that transfers some funds to an address controlled by the attacker. The attacker then waits for the transaction to be broadcast in the network. Once the attacker has received a copy of the transaction, the transaction is then modified using one of the above ways to alter the signature without invalidating it. The modification results in a different transaction identification hash. The modified transaction is then also broadcast in the network. Either of the two transactions may later be confirmed.

A malleability attack is said to be successful if the modified version of the transaction is later confirmed. The mechanics of how transactions are confirmed are complex and are out of scope for this work. For our purposes it suffices to say that the probability of a malleability attack to be successful depends on the distribution of nodes in the Bitcoin network first seeing either of the transactions (cf. 3 5]). So far the attack has not caused any damage to the victim. To be exploitable the victim also has to rely solely on the transaction identity hash to track and verify its account balance. Should a malleability attack be successful the victim will only see that the transaction it issued has not been confirmed, crediting the amount to the attacker or attempting to send another transaction at a later time. The attacker would have effectively doubled the bitcoins the victim sent it.

It is worth noting that the reference client (Bitcoin Core) is not susceptible to this attack as it tracks the unspent transaction output set by applying all confirmed transactions to it, rather than inferring only from transactions it issued. 


\section{MtGox Incident Timeline}

In this section we briefly describe the timeline of the incident that eventually led to the filing for bankruptcy of MtGox. The timeline is reconstructed from a series of press release by MtGox as well as the official filings and legal documents following the closure.

Following several months of problems with Bitcoin withdrawals from users, MtGox announced [6] on February 7 that it would suspend bitcoin withdrawals altogether. The main problem with withdrawals was that the associated Bitcoin transactions would not be confirmed. After this press release it was still possible to trade bitcoins on MtGox, but it was not possible to withdraw any bitcoins from the exchange. Specifically [6] does not mention transaction malleability.

In order to trade on MtGox, users had transferred bitcoins and US dollars to accounts owned by MtGox. Each user would have a virtual account that is credited with the transferred amounts at MtGox. The withdrawal stop therefore denied users access to their own bitcoins. While fiat currency was still withdrawable, such a withdrawal involved a long process that would sometimes fail altogether.

The first press release was followed by a second press release [7] on February 10, 2014. This press release claims that the problem for the non-confirming withdrawal transactions has been identified and names transaction malleability as the sole cause:

"Addressing Transaction Malleability: MtGox has detected unusual activity on its Bitcoin wallets and performed investigations during the past weeks. This confirmed the presence of transactions which need to be examined more closely.

Non-technical Explanation: A bug in the bitcoin software makes it possible for someone to use the Bitcoin network to alter transaction details to make it seem like a sending of bitcoins to a bitcoin wallet did not occur when in fact it did occur. Since the transaction appears as if it has not proceeded correctly, the bitcoins may be resent. MtGox is working with the Bitcoin core development team and others to mitigate this issue."

Allegedly a user of MtGox would request a withdrawal and listen for the resulting transaction. The transaction would then be intercepted and replaced by a modified version that would then race with the original transaction to be confirmed. Should the original transaction be confirmed, the user would receive its balance only once, but not lose any bitcoins by doing so. Should the modified transaction be confirmed, then the user would receive the bitcoins twice: once via the modified withdrawal transaction and a second time when MtGox realized that the original withdrawal transaction would not confirm and credit the users account. Implicitly in this press release MtGox admits to using a custom client that tracks transaction validity only via its hash, hence being vulnerable to the transaction malleability attack.

Two more press releases followed on February 17 and February 20, both claiming that the withdrawals would resume shortly and that a solution had been 
found that would eliminate the vulnerability to malleability attacks. On February 23 the website of MtGox returned only a blank page, without any further explanation, resulting in a trading halt and the complete disappearance of MtGox. Finally on February 28 MtGox announced during a press conference that it would be filing for bankruptcy in Japan and in the USA [8, 9].

\section{Measurements}

Due to the nature of double spending attacks, they may only be detected while participating in the network. As soon as one of the two conflicting transactions is considered to be confirmed the nodes will drop all other conflicting transactions, losing all information about the double spending attack. Malleability attacks being a subset of double spending attacks suffer from the same limitation.

We created specialized nodes that would trace and dump all transactions and blocks from the Bitcoin network. These include all double spending attacks that have been forwarded to any of the peers our nodes connected to. Our collection of transactions started in January 2013. As such we are unable to reproduce any attacks before January 2013. The following observations therefore do not consider attacks that may have happened before our collection started.

Our nodes were instructed to keep connection pools of 1,000 connections open to peers in the Bitcoin network. On average we connected to 992 peers, which at the time of writing is approximately $20 \%$ of the reachable nodes. According to Bamert et al. 3] the probability of detecting a double spending attack quickly converges to 1 as the number of sampled peers increases. We therefore feel justified in assuming that the transactions collected during the measurements faithfully reflect the double spending attacks in the network during the same period.

\subsection{Global Analysis}

Given the set of all transactions, the first task is to extract all potential double spend attacks. In general double spending attacks can be identified by associating a transaction with each output that it claims. Should there be more than one transaction associated with the same output the transactions conflict. The malleability attack being a specialized case of the double spend attack could also be identified by this generic procedure, however we opted for a simpler process. Removing the signature script from a transaction results in the signed part of the transaction, forcing all malleability attacks to produce the same unique key. The unique key is then used to group transactions together into conflict sets.

During the measurement period a total of 35,202 conflict sets were identified, each evidence of a malleability attack. Out of these conflict sets 29,139 contained a transaction that would later be confirmed by a block. The remaining 6,063 transactions were either invalid because they claimed non-existing outputs, had incorrect signatures, or they were part of a further double spending.

The conflict set value is defined as the number of bitcoins transferred by any one transaction in the conflict set. The outputs of the transactions in a 
conflict set are identical, since any change to them would require a new signature. In particular the value of outputs may not be changed. Each transaction in a conflict set therefore transfers an identical amount of bitcoins. Summing the value of all conflict sets results in a total of 302,700 bitcoins that were involved in malleability attacks.

As mentioned in Section 2.1, there are a multitude of ways to use the malleability in the signature encoding to mount a malleability attack. The most prominent type of modification was replacing the single byte OP_0 with OP_PUSHDATA2 which then encodes the length of the data to push on the stack with 2 bytes. The resulting signature script would be 4 bytes longer, because two strings are usually pushed on the stack, but would still encode the same DER encoded signature and the same public key, hence still be valid. A total of 28,595 out of the 29,139 confirmed attacks had this type of modifications. For the remaining 544 conflict sets we were unable to identify the original transactions. All transactions in these conflict sets had genuine signatures with the correct opcodes and did not encode the same signature. We therefore believe these transactions to be the result of users signing raw transactions multiple times, e.g., for development purposes.

In order for a malleability attack to be exploitable two conditions have to be fulfilled: (a) the modified transaction has to be later confirmed and (b) the system issuing the transaction must rely solely on the transaction's original hash to track its confirmation. The first condition can be easily reconstructed from the network trace and the Bitcoin blockchain since only one of the transactions will be included in the blockchain. The second condition is not detectable in our traces since it depends on the implementation of the issuing system. In particular, it is not possible to determine whether two payments with the same value to the same address were intended as two separate payments or whether an automated system issued the second one believing the first to be invalid.

We call a malleability attack successful if it resulted in the modified transaction to be later confirmed in a block, i.e., when condition (a) holds. From the data derived from the attack classification we can measure the rate of successful malleability attacks. Out of the 28,595 malleability attacks that used an OP_PUSHDATA2 instead of the default OP_0 only 5,670 were successful, i.e., $19.46 \%$ of modified transactions were later confirmed. Considering the value in malleable transactions the success rate is comparable with $21.36 \%$. This reduces the total profit of the successful attacks from 302,700 to 64,564 . The strong bias towards the original transaction is explained by the fact that the probability of being confirmed depends on the distribution of the transaction in the network [3]. During a malleability attack the attacker listens for an incoming transaction that match its address, modifies it and redistributes it. In the meantime however the original transaction has been further forwarded in the network and the modified transaction is not forwarded by nodes seeing the original transaction. The attacker must connect to a large sample of nodes in the network for two reasons: (a) intercept the original transaction as soon as possible and (b) 

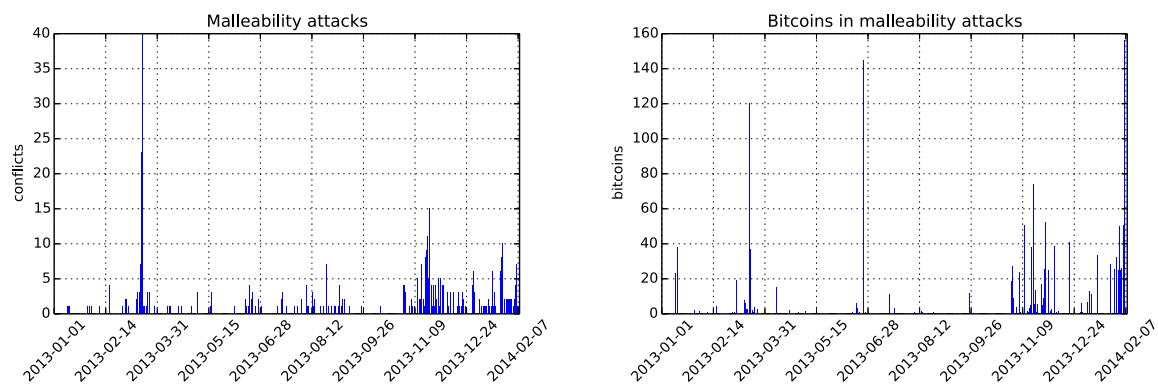

Fig. 2. Malleability attacks during period 1, before the press release blaming transaction malleability as the sole cause of losses

compensate the head start that the original transaction has compared to the modified transaction.

So far we assumed that the conflict sets were a direct result of a targeted attack by an attacker against a service. There are however other causes for this kind of conflict that should not go unmentioned. An automated system may inadvertently create, sign a transaction and broadcast a transaction multiple times. Due to a random parameter in the signing process the system would produce a different signature each time, causing the conflict that we detected. This appears to be the case with transactions having conflict set cardinality larger than 2 , that would often not be confirmed.

\subsection{The MtGox Incident}

Returning to the specific case of the MtGox incident of February 2014, that eventually lead to the closure and the bankruptcy filing later that same month. In the press release of February 10, the transaction malleability bug was explicitly named as the root cause of the loss. The loss is later detailed as amounting to over 850,000 bitcoins, of which 750,000 bitcoins were customer owned bitcoins that were managed by MtGox. At the time of the first press release bitcoins were trading at 827 US Dollars per bitcoin 2 resulting in a total value of lost bitcoins of 620 million US Dollars.

Assuming malleability attacks have indeed been used to defraud MtGox, then we should be able to verify the claim by finding the transactions used for the attack in our dataset. The above mentioned total amount of 302,700 bitcoins involved in malleability attacks already disproves the existence of such a large scale attack. However, it could well be that malleability attacks contributed considerably in the declared losses.

Reconstructing the timeline of the attacks from the announcements made by MtGox we identify 3 time periods:

\footnotetext{
${ }^{2}$ Exchange rate taken as the open value on MtGox of February 7, 2014.
} 
- Period 1 (January 2013 — February 7, 2014): over a year of measurements until the closure of withdrawals from MtGox;

- Period 2 (February 8 - February 9, 2014): withdrawals are stopped but no details about the attack known to the public;

- Period 3 (February 10 - February 28): time following the press release blaming transaction malleability as the root cause of the missing bitcoins until MtGox filed for bankruptcy.

Malleability attacks in period 2 and 3 could not contribute to the losses declared by MtGox since they happened after withdrawals have been stopped. Figure 2 visualizes both the number of bitcoins involved in malleability attacks as well as the number of attacks during period 1. During this period a total of 421 conflict sets were identified for a total value of $1,811.58$ bitcoins involved in these attacks. In combination with the above mentioned success rate of malleability attacks we conclude that overall malleability attacks did not have any substantial influence in the loss of bitcoins incurred by MtGox.

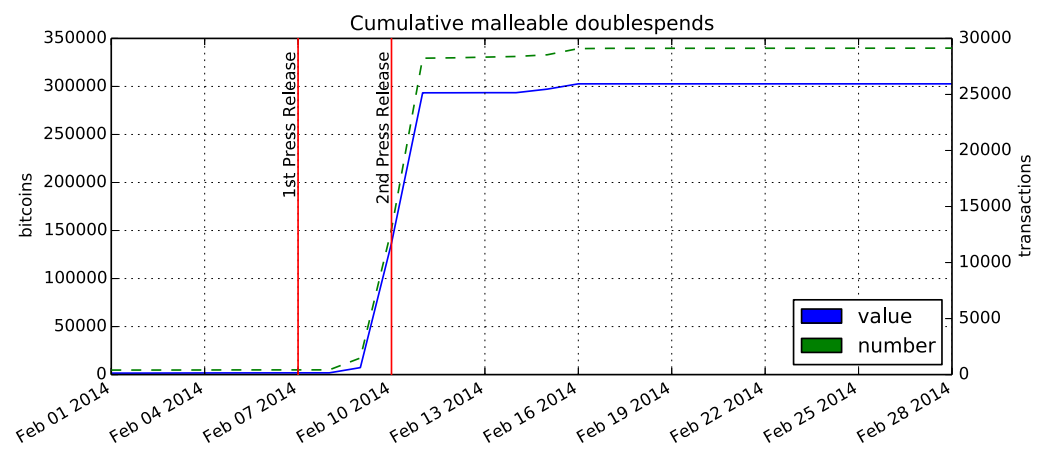

Fig. 3. Cumulative graph of the number and value of malleability attacks during the time of the press releases

During period 2, we gathered 1,062 conflict sets, totalling 5,470 bitcoins. A noticeable increase of attacks at 17:00 UTC on February 9, from 0.15 attacks per hour to 132 attacks per hour. While we do not have any information about the time the second press release has been published, the measured increase in attacks at 17:00 UTC and the date on the press release, hints at a time between 0:00 and 2:00 JST. The sudden increase suggests that immediately following the press release other attackers started imitating the attack, attempting to exploit the same weakness that had allegedly been used against MtGox.

After the second press release, in period 3, there is a sudden spike in activity. Between February 10 and 11 we identified 25,752 individual attacks totalling 286,076 bitcoins, two orders of magnitude larger than all attacks from period 1 combined. A second, smaller, wave of attacks starts after February 15, with a 
total of 9,193 bitcoins. The attacks have since calmed, returning to levels comparable to those observed in period 1 , before the press releases. Figure 3 summarizes the situation plotting the cumulative value and number of malleability attacks in February 2014, i.e., from the end of period 1 to period 3.

The strong correlation between the press releases and the ensuing attacks attempting to exploit the same weakness is a strong indicator that the attacks were indeed triggered by the press releases.

Assuming MtGox had disabled withdrawals like they stated in the first press release, these attacks can not have been aimed at MtGox. The attacks therefore where either attempts to investigate transaction malleability or they were aimed at other businesses attempting to imitate the purveyed attack for personal gain. The sheer amount of bitcoins involved in malleability attacks would suggest that the latter motive was prevalent.

It remains questionable whether other services have been informed by MtGox in time to brace for the sudden increase in malleability attacks. Should this not be the case then the press release may have harmed other businesses by triggering imitators to attack them.

\subsection{Beyond Our Data}

In the previous subsections we presented an analysis of malleability attacks based on data we collected for over a year preceding the bankruptcy filing by MtGox. We have limited the analysis to the timespan we have first-hand data, starting January 2013. Clearly attacks may have happened even before our measurements started. However, in our opinion, it is unlikely that transaction malleability was exploited on a large scale before our measurements, and not during our measurements. After all, why would an attacker, having found such a lucrative attack before 2013, suddenly stop exploiting it? It seems more likely that an attacker would use this risk-free and successful attack more often and with larger amounts of bitcoins!

While it is not possible to detect all malleability attacks without participating in the network at the time they occur, we can estimate the number of attacks preceding our measurements, just by reading the blockchain. By far the most common modification during our measurements was the use of non-minimal push opcodes, over $98 \%$ out of all attacks use this modification. Successful attacks, i.e., those that were eventually confirmed, can be found by searching for this modification in the set of all confirmed transactions. Given the success rate and the number of successful attacks we can extrapolate the number of attacks that were attempted before our measurements began.

By inspecting all confirmed transactions for signature scripts that do not use minimal push opcodes we found a total of 48 transactions, involving a total of 33.92 bitcoins, before our measurements started, i.e., in the period 2009 2012. Assuming that the success rate of $21.34 \%$ did not change significantly, we can extrapolate a total of less than 160 bitcoins involved in a few hundreds of attempted malleability attacks preceding our measurements. This is equivalent to less than $10 \%$ of the attacks identified during our measurements. 
Besides the temporal restriction of our study, we also restricted ourselves to one specific attack, made possible by transaction malleability. Malleability attacks as defined in Section 2.2 require that both the original and the modified transaction are broadcast in the Bitcoin network. This reflects the description of the attack in the MtGox press release of February 10, 2014 [7].

In addition to broadcasting the transactions in the network, MtGox also published withdrawal transactions on their website. This may have resulted in a different attack, only partially covered by this work. MtGox sometimes created invalid transactions with non-canonical signatures which would not be forwarded by newer Bitcoin clients. An attacker could retrieve the invalid transactions, correct the signatures and release the corrected transactions into the network.

We were able to collect these invalid transactions until October 2013, but not after that. The collected invalid transactions were considered when creating the conflict sets and figures in the analysis. It is however possible that some transactions did not even reach the Bitcoin network, and that some different type of attack might have played a role in MtGox' loss. We would like to stress that this paper does focus on malleability attacks only, as defined by MtGox and in this paper. Other types of attacks are outside the scope of this paper.

Finally, it is worth noting that the attacks described in this work could have been countered by adhering to basic best practices. Failed transactions should not be automatically retried, since a failure to confirm is indicative of other errors, as would have been the case with non-canonical signatures or malleability attacks. Should automatic retrial be desired, then the transaction issuer must ensure that the same inputs are reused. By doing so the issuer ensures that the funds are transferred at most once, even if an attacker may arbitrarily delay the transaction or exploit transaction malleability to render the original transaction unrecognizable.

\section{Related Work}

Transaction malleability has been known about since at least 2010, when it was first documented. It has however received very little attention so far as it was categorized as a low priority issue.

Andrychowicz et al. [10, 11] mention transaction malleability as a potential problem in contracts and two party computations based on Bitcoin transactions. These schemes can be used for example to implement a fair coin toss [12], auctions or decentralized voting. Their method to eliminate transaction malleability in their protocols resembles our construction of conflict sets, i.e., eliminating malleable parts of the transaction in the hash calculation. However, they limit their observations to advanced schemes for encoding contracts and two party computations.

A related class of doublespending attacks, which we shall refer to as classical doublespending, has received far more attention. In this class of attacks the transaction issuer creates two transactions to defraud the receiving party. Karame et al. 5] first studied the problem of arising from fast transactions, 
i.e., accepting non-confirmed transactions. Rosenfeld [13] showed that the success probability of a doublespending attack can be further increased if coupled with computational resources. Bamert et al. 3] later improved the security of accepting fast payments by observing how transactions are propagated in the network.

To the best of our knowledge this paper is the first publication describing transaction malleability and the resulting malleability attack in detail.

\section{Conclusion}

The transaction malleability problem is real and should be considered when implementing Bitcoin clients. However, while MtGox claimed to have lost 850,000 bitcoins due to malleability attacks, we merely observed a total of 302,000 bitcoins ever being involved in malleability attacks. Of these, only 1,811 bitcoins were in attacks before MtGox stopped users from withdrawing bitcoins. Even more, $78.64 \%$ of these attacks were ineffective. As such, barely 386 bitcoins could have been stolen using malleability attacks from MtGox or from other businesses. Even if all of these attacks were targeted against MtGox, MtGox needs to explain the whereabouts of 849,600 bitcoins.

\section{References}

1. Nakamoto, S.: Bitcoin: A peer-to-peer electronic cash system, https://bitcoin.org/bitcoin.pdf (Online; accessed March 26, 2014)

2. Wuille, P.: BIP 0062: Dealing with Malleability (2014), https://github.com/bitcoin/bips (Online; accessed March 10, 2014)

3. Bamert, T., Decker, C., Elsen, L., Welten, S., Wattenhofer, R.: Have a snack, pay with bitcoin. In: IEEE Internation Conference on Peer-to-Peer Computing (P2P), Trento, Italy (2013)

4. Decker, C., Wattenhofer, R.: Information propagation in the bitcoin network. In: IEEE International Conference on Peer-to-Peer Computing (P2P), Trento, Italy (September 2013)

5. Karame, G., Androulaki, E., Capkun, S.: Two Bitcoins at the Price of One? DoubleSpending Attacks on Fast Payments in Bitcoin. In: Proc. of Conference on Computer and Communication Security (2012)

6. MtGox: Mtgox press release announcing the stop of withdrawals (2014), https://www.mtgox.com/press_release_20140210.html

(Online; accessed February 10, 2014)

7. MtGox: Mtgox press release about transaction malleability (2014), https://www. mtgox.com/press_release_20140210.html (Online; accessed February 10, 2014)

8. MtGox: Announcement regarding an application for commencement of a prodedure of civil rehabilitation, https://www.mtgox.com/img/pdf/20140228announcement_eng.pdf (Online; accessed March 19)

9. MtGox: Announcement regarding the applicability of us bankruptcy code chapter 15, https://www.mtgox.com/img/pdf/20140314-announcement_chapter15.pdf (Online; accessed March 19) 
10. Andrychowicz, M., Dziembowski, S., Malinowski, D., Mazurek, Ł.: Fair two-party computations via the bitcoin deposits. Technical report, Cryptology ePrint Archive (2013)

11. Andrychowicz, M., Dziembowski, S., Malinowski, D., Mazurek, Ł.: How to deal with malleability of bitcoin transactions. arXiv preprint arXiv:1312.3230 (2013)

12. Back, A., Bentov, I.: Note on fair coin toss via bitcoin. arXiv preprint arXiv:1402.3698 (2014)

13. Rosenfeld, M.: Analysis of hashrate-based double spending (2012), https://bitcoil.co.il/Doublespend.pdf (Online; accessed February 17, 2014) 